CORRIGENDUM

doi:10.1038/nature12527

\title{
Corrigendum: $\mathrm{X}$-ray analysis on the nanogram to microgram scale using porous complexes
}

Yasuhide Inokuma, Shota Yoshioka, Junko Ariyoshi, Tatsuhiko Arai, Yuki Hitora, Kentaro Takada,

Shigeki Matsunaga, Kari Rissanen \& Makoto Fujita

Nature 495, 461-466 (2013); doi:10.1038/nature11990

Previously unnoticed ambiguities in the crystallographic data in our Article (specifically, non-negligible disorder of the miyakosyne A molecule), along with further work by three of the authors (Y.H., K.T. and S.M.) have revealed that the stereochemistry we assigned at C14 of miyakosyne A is incorrect. Our new investigations confirm that we can indeed determine the molecular skeleton of miyakosyne A. However, we can only tentatively and not unambiguously identify all of the stereochemistry of miyakosyne A based on the data included in the original paper. A future publication will confirm the stereochemistry at the $\mathrm{C} 14$ moiety of miyakosyne A. The other conclusions of our paper are not affected by this correction. 